\title{
As contribuições da medicina antroposófica ao processo de empoderamento e co-criação da saúde da mulher: revisão integrativa
}

\section{The contributions of anthroposophic medicine to the process of empowerment and co-creation of women's health: integrative review}

DOI: 10.54022/shsv3n1-006

Recebimento dos originais: 05/12/2021

Aceitação para publicação: 05/01/2022

\section{Débora Regina Buono Gontow}

Débora Regina Buono Gontow - Graduanda em Medicina da Universidade do Vale do Rio dos Sinos - UNISINOS/RS - Rua Anita Garibaldi 1122/806 - Porto Alegre/RS

E-mail: deboragontow@gmail.com

\section{Rosália Figueiró Borges}

Doutora em Educação - Docente da graduação e da Pós-graduação em Enfermagem da Universidade do Vale do Rio dos Sinos - UNISINOS/RS - Rua Guarau, 53 - Novo Hamburgo/RS;

E-mail: rosaliafb@unisinos.br;

\section{Ana Paula Machado Scienza}

Mestranda em Enfermagem Profissional do Programa de Pós-graduação em Enfermagem da Universidade do Vale do Rio dos Sinos - UNISINOS/RS -

Rua Júlio de Castilhos, número 30 - São Leopoldo/RS

E-mail: pmscienza@gmail.com

\section{Carine Schneider de Lima}

Mestranda em Enfermagem Profissional do Programa de Pós-graduação em Enfermagem da Universidade do Vale do Rio dos Sinos - UNISINOS/RS

- Enfermeira Assistencial Unidade de Terapia Intensiva - UTI Adulto do Hospital Montenegro 100\% SUS - Montenegro/RS - Av. Pedro Chies, 657/302 - São Pedro da Serra/RS;

E-mail: carinesl22@yahoo.com.br

\section{Edileuza Nunes dos Santos}

Mestranda em Enfermagem Profissional do Programa de Pós Graduação em Enfermagem da Universidade do Vale do Rio dos Sinos - UNISINOS/RS

- Enfermeira de Medicina Preventiva na Unimed Encosta da Serra - Taquara/RS

- Rua Julio de Castilhos, 2400/31 - Taquara/RS;

E-mail: edileuzanunes90@gmail.com 


\section{Maine Serena Pasa}

Graduanda em Medicina da Universidade do Vale do Rio dos Sinos UNISINOS/RS - Rua Aloísio Haubert, 275 - São Leopoldo/RS;

E-mail: mainespasa@gmail.com

\section{Mariani Mello da Silva}

Mestranda em Enfermagem Profissional do Programa de Pós Graduação em

Enfermagem da Universidade do Vale do Rio dos Sinos - UNISINOS/RS

- Enfermeira Auditora do Exército Brasileiro do Comando da 3a Região Militar -

Porto Alegre/RS - Av. Padre Cacique, 50/416 - Porto Alegre/RS;

E-mail:mariani.mellos@gmail.com

\section{Resumo}

As Medicinas Tradicionais Complementares e Integrativas (MTCI) são definidas como um arcabouço de abordagens em saúde, que contemplam o ser em sua integralidade e transpõem as barreiras entre a mente, o corpo e o espírito. No campo da saúde, analisa-se a manutenção de um modelo biomédico hegemônico, que é caracterizado pela centralização na biologia humana, na medicalização social, na fragmentação do cuidado e na assimetria das relações curadorpaciente. O conservadorismo na formação dos profissionais de ginecologia obstétrica, o atrelamento da mulher à função reprodutiva, a regulação da sexualidade e a patologização dos corpos femininos são implicações destas normas biomédicas e, em meio à mercantilização e a impessoalidade dos serviços médicos, a mulher se viu despida de todo conhecimento sobre sua própria saúde. A Medicina Antroposófica rompe o paradigma da terceirização da saúde, ao propor o diálogo que integra as racionalidades médicas e, sobretudo, empoderando a mulher à participação ativa e ao protagonismo do processo de cocriação da sua própria saúde. Diante deste contexto, desenvolveu-se a questão de pesquisa: o que diz a literatura sobre a Medicina Antroposófica no processo de empoderamento e autonomização da saúde da mulher? Pretendeu-se analisar a produção científica referente aos principais benefícios da Medicina Antroposófica e as suas contribuições acerca da integralidade da saúde da mulher. Trata-se de um estudo qualitativo, descritivo e exploratório com ênfase em revisão integrativa da literatura. A coleta de dados, realizada por meio de uma consulta às bases de dados eletrônica da Biblioteca Virtual em Saúde (BVS) e MTCI Américas no mês de julho de 2020, evidenciou um total de 35 artigos e após aplicar os critérios de inclusão e exclusão, obteve-se uma amostra de 25 artigos. Foi realizada a leitura dos resumos, totalizando uma amostra de 21 artigos, que foram lidos na íntegra, com utilização do sistema de nível de evidências. Os resultados das publicações foram abordados a partir do agrupamento em cinco categorias definidas a priori: câncer de mama, doenças crônicas e psicossomáticas, saúde da mulher, obstetrícia e sistemas de saúde. As evidências científicas sobre o processo de empoderamento e autonomização da saúde da mulher destacam que a Medicina Antroposófica se revela como uma ferramenta ativa de mudança no paradigma da doença à potencialização da saúde e caracteriza-se, portanto, como uma prática que contribui para o fortalecimento da autonomia e determinação da mulher nos seu processo de saúde-doença nas diferentes fases do ciclo vital.

Palavras-Chave: Terapias Complementares. Antroposofia. Saúde da Mulher. Empoderamento. 


\section{ABSTRACT}

Traditional Complementary and Integrative Medicines (TCMIs) are defined as a framework of approaches to health, which contemplate the whole being and overcome the barriers between mind, body, and spirit. In the health field, the maintenance of a hegemonic biomedical model is analyzed, which is characterized by the centralization in human biology, in social medicalization, in the fragmentation of care, and in the asymmetry of the healer-patient relationship. The conservatism in the training of obstetric gynecology professionals, the attachment of women to the reproductive function, the regulation of sexuality, and the pathologization of female bodies are implications of these biomedical norms and, in the midst of the commercialization and impersonality of medical services, women were stripped of all knowledge about their own health. Anthroposophic Medicine breaks the paradigm of health outsourcing by proposing a dialogue that integrates medical rationalities and, above all, by empowering women to active participation and protagonism in the process of co-creation of their own health. In this context, the research question was developed: what does the literature say about Anthroposophic Medicine in the process of empowerment and autonomy of women's health? The objective was to analyze the scientific production referring to the main benefits of Anthroposophic Medicine and its contributions to the integrality of women's health. This is a qualitative, descriptive and exploratory study with emphasis on an integrative literature review. Data collection, carried out by means of a query to the electronic databases of the Virtual Health Library (VHL) and MTCI Americas in the month of July 2020, revealed a total of 35 articles, and after applying the inclusion and exclusion criteria, a sample of 25 articles was obtained. The abstracts were read, totaling a sample of 21 articles, which were read in full, using the evidence level system. The results of the publications were approached from the grouping into five categories defined a priori: breast cancer, chronic and psychosomatic diseases, women's health, obstetrics, and health systems. The scientific evidence on the process of empowerment and autonomy of women's health highlights that Anthroposophic Medicine reveals itself as an active tool for change in the paradigm of the disease to the potentialization of health and is characterized, therefore, as a practice that contributes to strengthening the autonomy and determination of women in their health-disease process in the different stages of the life cycle.

Keywords: Complementary Therapies, Anthroposophy, Women's Health, Empowerment.

\section{INTRODUÇÃO}

As Medicinas Tradicionais Complementares e Integrativas (MTCI) são definidas como um arcabouço de abordagens em saúde, que contemplam o ser em sua integralidade e transpõem as barreiras entre a mente, o corpo e o espírito. (ANDRADE; COSTA, 2010). Ressalta-se, que ocorreu uma evolução dos anos 90 à atualidade, no que tange ao reconhecimento e a difusão das Práticas Integrativas e Complementares em Saúde (PICS) em proporções mundiais. Tal 
crescimento e visibilidade decorrem principalmente pelo estímulo da OMS que desde 2002 tem realizado iniciativas por meio da elaboração de um documento normativo para seus países membros. (PROGEPE, 2021).

As PICS, foram institucionalizadas no Brasil e ofertadas no âmbito da saúde pública brasileira conforme a Portaria interministerial 971, que, em 2006, instituiu a Política Nacional de Práticas Integrativas e Complementares (PNPIC). Esta política assegurou e promoveu o acesso, no Sistema Único de Saúde (SUS), à medicina tradicional chinesa, à homeopatia, à fitoterapia, ao termalismo e à medicina antroposófica (BRASIL, 2006). Esta publicação da PNPIC, mobilizou Estados e municípios a produzirem suas próprias diretrizes políticas relativas à oferta e ao uso das PICS no SUS. Em 2015, publicou-se a segunda edição acerca da oferta e o estímulo das PICS como a fitoterapia, a homeopatia, acupuntura, dentre outras, foi legitimada pelo SUS e ampliando sua utilização. (PROGEPE, 2021).

Neste contexto, o Brasil se destaca como um dos 69 membros da OMS que possuem políticas e estratégias específicas para o uso das PICS. Após a criação da PNPIC, houve um aumento de $30 \%$ dos municípios brasileiros adotaram regulamentação para o uso das terapias, indicando um incremento importante das PICS na atenção à saúde.

No ano de 2017, as PICS tornaram-se oficiais perante o Ministério da Saúde (MS), que reconheceu a importância das manifestações populares em saúde e a chamada medicina não convencional, considerada como prática voltada à saúde e ao equilíbrio vital do homem. (PROGEPE, 2021). A portaria $\mathrm{n}^{\circ} \mathbf{8 4 9}$, de 27 de março de 2017, legitimou 14 novas práticas e, em 2018, mais 10 práticas com a portaria no 702, de 21 de março de 2018 (PESSOA et al., 2021).

A evolução do paradigma sociocultural-histórico fez com que o mundo enfrentasse desafios impostos pela globalização e as consequentes transformações sociais, e, no campo da saúde, analisa-se a manutenção de um modelo biomédico hegemônico, que é caracterizado pela centralização na biologia humana, na medicalização social, na fragmentação do cuidado e na assimetria das relações curador-paciente. (BASTOS; PEREIRA, 2018; TESSER; BARROS, 2008).

Neste sentido, as implicações destas normas biomédicas no contexto da 
saúde da mulher se deram de diversas formas: o conservadorismo na formação dos profissionais de ginecologia obstétrica, o atrelamento da mulher à função reprodutiva, a regulação da sexualidade e a patologização dos corpos femininos. (BASTOS; PEREIRA, 2018; AGUIAR; D'OLIVEIRA, 2010).

Destaca-se que, em meio à mercantilização e a impessoalidade dos serviços médicos, a mulher se viu despida de todo conhecimento sobre sua própria saúde. Assim sendo, a Medicina Antroposófica rompe o paradigma da terceirização da saúde, ao propor o diálogo que integra as racionalidades médicas e, sobretudo empoderando a mulher à participação ativa e ao protagonismo do processo de co-criação da sua própria saúde. (BASTOS; PEREIRA, 2018; BORGES; MADEIRA; AZEVEDO, 2011).

Diante deste contexto, desenvolveu-se a questão de pesquisa deste estudo: o que diz a literatura sobre a Medicina Antroposófica no processo de empoderamento e autonomização da saúde da mulher? Para responder a este questionamento, pretendeu-se analisar a produção científica referente aos principais benefícios da Medicina Antroposófica e as suas contribuições acerca da integralidade da saúde da mulher.

\section{METODOLOGIA}

Trata-se de um estudo qualitativo, descritivo e exploratório com ênfase em revisão integrativa da literatura. O propósito da revisão integrativa de literatura é sintetizar resultados sobre determinado assunto com informações amplas. Possui seis etapas: formulação da pergunta de revisão (orienta o foco do estudo), amostragem (determina os estudos relevantes - é a etapa onde são estabelecidos os critérios de inclusão e exclusão do estudo, identificação dos descritores, busca nas bases de dados e seleção dos artigos), realização de leitura na íntegra para extrair os principais resultados, avaliação crítica dos resultados obtidos, neste caso, com utilização do sistema de nível de evidências. (LACERDA; COSTENARO, 2016).

A estratégia PICO (acrônimo para population, intervention, comparison and outcome) foi utilizada para elaboração da questão norteadora, com a finalidade de descobrir as melhores evidências científicas. Para formular o acrônimo PICO considerou-se "saúde da mulher" como o P (Patient or problem), 
"quais os benefícios da medicina antroposófica para a integralidade do cuidado na atenção à saúde da mulher" como I (Intervention), para o C (Comparation) não houve aplicação e, "contribuições da Medicina Antroposófica na saúde na mulher" foi utilizado para o $\mathbf{O}$ (Outcome).

A coleta dos artigos foi realizada por meio de uma consulta às bases de dados eletrônica da Biblioteca Virtual em Saúde (BVS) e MTCI Américas no mês de julho de 2020. Foram utilizados os descritores contemplados no MeSH (Medical Subject Headings) e os operadores "OR" e "AND", resultando na combinação "Anthroposophic" AND "Women's Health" OR "Feminine". Foram incluídos artigos publicados no período de 2006 a 2019, nos idiomas português e inglês. Foram excluídas publicações que não apresentavam texto na íntegra, estudos que não abordassem a temática da pesquisa ou que estivessem apresentadas em formato de tese, dissertações, manuais, capítulos de livros, reflexões e editais. Os materiais foram lidos na totalidade e analisados de acordo com a pergunta PICO. Conforme Lacerda e Costenaro (2016), após a seleção dos artigos, os mesmos foram sequenciados em um quadro sinóptico contendo informações como: título, autor, ano, país e nível de evidência. Definiu-se à priori três categorias: terapias complementares; saúde da mulher e promoção de saúde. Os artigos repetidos foram considerados uma única vez. Juntamente com as outras informações, o quadro sinóptico contempla a classificação de acordo com o grau de evidência proposto por Melnyk e Fineout- Overhlt. (2011). A classificação de evidências é o alicerce que determina o nível de confiança do estudo, bem como o uso dos seus resultados.

Esta revisão integrativa fundamentou-se na Resolução № 510/2016 do Conselho Nacional de Saúde, no parágrafo único, inciso VI, a qual discorre sobre pesquisa realizada exclusivamente com textos científicos para revisão da literatura científica e que não serão registradas nem avaliadas pelo sistema CEP/CONEP. Além disso, seguiu-se os preceitos das Leis n 9.610, de 19 de fevereiro de 1998 e no 12.853 de 14 de agosto de 2013 que dispõem sobre gestão coletiva de direitos autorais. 


\section{RESULTADOS}

A coleta evidenciou um total de 35 artigos e após aplicar os critérios de inclusão, obteve-se uma amostra de 25 artigos. Foi realizada a leitura dos resumos e, sendo excluídos os documentos que não apresentavam tanto o texto completo, quanto a abordagem da relação entre a medicina antroposófica e a saúde da mulher, totalizou uma amostra de 21 artigos, conforme apresentado na figura 1 - fluxograma das etapas de seleção dos artigos.

Figura 1- Fluxograma das etapas de seleção dos artigos

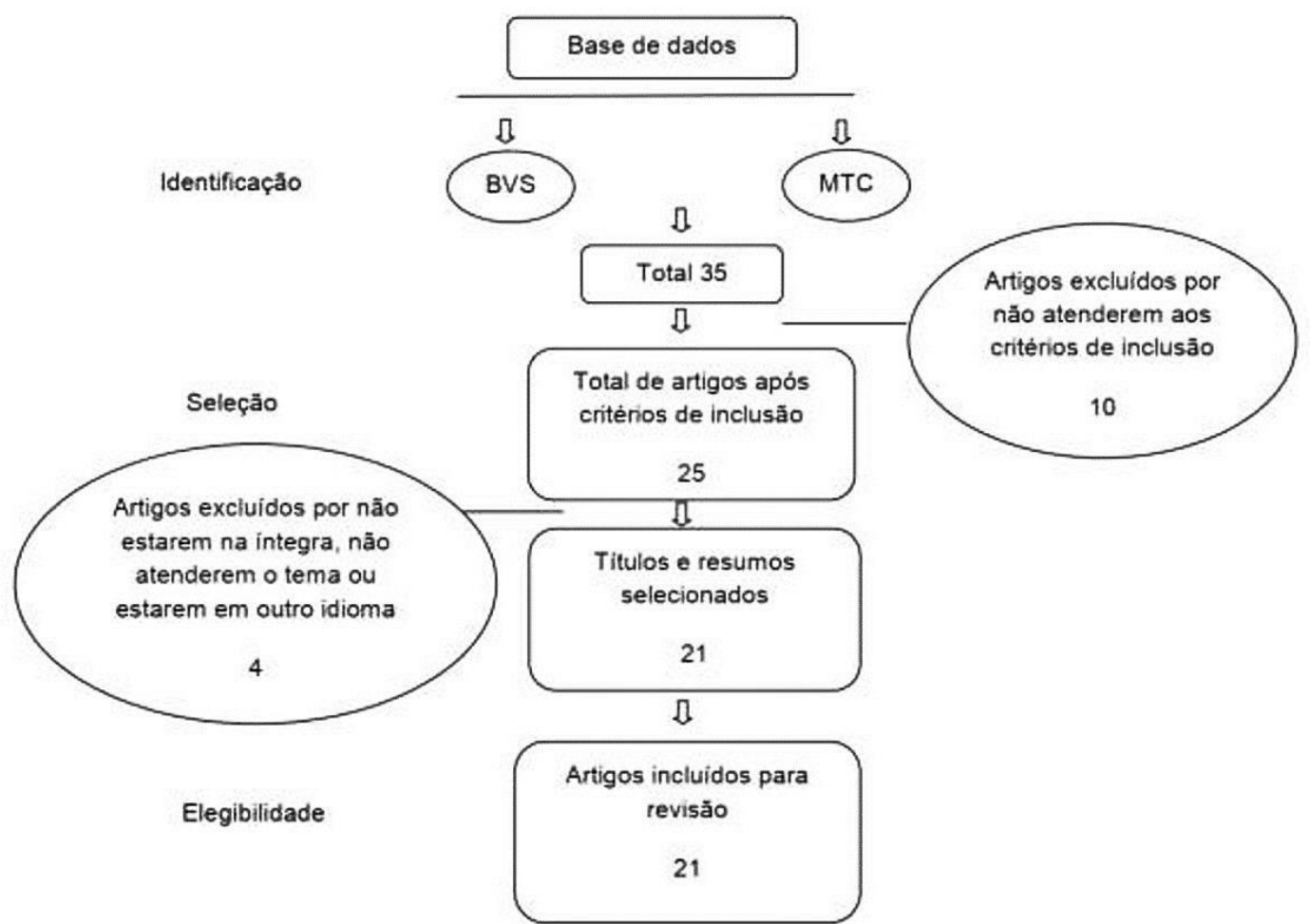

Fonte: elaborado pelas autoras (2022).

Analisou-se 21 artigos na íntegra, que atenderam aos critérios de inclusão pré-estabelecidos. Com relação ao período de publicação dos artigos, oito (38,0 \%) representam 1 artigo em cada ano de: 2006, 2007, 2009, 2010, 2011, 2012, 2016 e 2017, três artigos (14,3\%) eram de 2014, 2018, 2019 e quatro artigos $(19,0 \%)$ eram de 2015.

Com relação aos tipos de delineamento de pesquisa dos artigos avaliados, 
evidenciou-se: nove (42,8\%) estudos de coorte, quatro (19\%) qualitativos, quatro (19\%) ensaios clínicos randomizados controlados, duas $(9,5 \%)$ revisões sistemáticas da literatura, além de um (4,7\%) estudo observacional e um $(4,7 \%)$ estudo multicêntrico.

A análise dos presentes artigos evidenciou que nove artigos (42,8\%) das publicações eram provenientes da Suíça, seis artigos (28,5\%) eram da Alemanha, cinco $(24,8 \%)$ eram originários da Suécia, três $(14,2 \%)$ do Brasil e dois estudos $(9,5 \%)$ eram da holanda.

Quanto ao nível de evidência, nove artigos (42,8\%) são do nível de evidência 4, quatro artigos (19\%) representaram nível de evidência 2, seis artigos (28,5\%) eram nível 6 e dois artigos (9,5\%) apresentaram nível de evidência 5.

Os resultados das publicações serão abordados a partir do agrupamento em cinco categorias definidas a priori, objetivando melhor organização das informações. As categorias foram: câncer de mama, doenças crônicas e psicossomáticas, saúde da mulher, obstetrícia e sistemas de saúde, conforme o quadro 1:

Quadro 1- Categoria dos Achados

\begin{tabular}{|c|c|c|c|c|c|}
\hline & Título do artigo & Autor & Ano & País & $\begin{array}{l}\text { Nível de } \\
\text { Evidênci } \\
\text { a }\end{array}$ \\
\hline & \multicolumn{5}{|c|}{ CATEGORIA 1- CÂNCER DE MAMA } \\
\hline A1 & $\begin{array}{l}\text { A Five-year Follow-up of Quality of Life } \\
\text { in Women with Breast Cancer in } \\
\text { Anthroposophic and Conventional Care }\end{array}$ & $\begin{array}{l}\text { CARLSS } \\
\text { ON et al. }\end{array}$ & 2006 & Suécia & N4 \\
\hline A2 & $\begin{array}{l}\text { A longitudinal study on women's } \\
\text { experiences of life with breast cancer in } \\
\text { anthroposophical (complementary) and } \\
\text { conventional care }\end{array}$ & $\begin{array}{l}\text { ARMAN; } \\
\text { BACKM } \\
\text { AN }\end{array}$ & 2007 & Suécia & N6 \\
\hline A3 & $\begin{array}{l}\text { Immunological and Quality-of-Life } \\
\text { Profiles in Women with Breast Cancer: } \\
\text { Complementary versus Conventional } \\
\text { Care }\end{array}$ & $\begin{array}{c}\text { HAMRIN } \\
; \\
\text { ERNER } \\
\text { UDH; } \\
\text { ROSÉN. }\end{array}$ & 2018 & Suécia & N4 \\
\hline
\end{tabular}




\begin{tabular}{|c|c|c|c|c|c|}
\hline & \multicolumn{5}{|c|}{ CATEGORIA 2- DOENÇAS CRÔNICAS, PSICOSSOMÁTICAS } \\
\hline A4 & $\begin{array}{l}\text { Outcome of anthroposophic medication } \\
\text { therapy in chronic disease: a 12-month } \\
\text { prospective cohort study. }\end{array}$ & $\begin{array}{l}\text { HAMRE } \\
\text { et al. }\end{array}$ & 2009 & $\begin{array}{l}\text { Alema } \\
\text { nha } \\
\text { Suíça }\end{array}$ & N4 \\
\hline A5 & $\begin{array}{l}\text { Anthroposophic therapy for migraine: a } \\
\text { two-year prospective cohort study in } \\
\text { routine outpatient settings. }\end{array}$ & $\begin{array}{l}\text { HAMRE } \\
\text { et al. }\end{array}$ & 2010 & $\begin{array}{l}\text { Alema } \\
\text { nha } \\
\text { Suíça }\end{array}$ & N4 \\
\hline A6 & $\begin{array}{l}\text { Anthroposophic health care in Sweden e } \\
\text { A patient evaluation }\end{array}$ & $\begin{array}{c}\text { ARMAN; } \\
\text { HAMMA } \\
\text { RQVIST; } \\
\text { KULLBE } \\
\text { RG. }\end{array}$ & 2011 & Suécia & N4 \\
\hline A7 & $\begin{array}{l}\text { Self-care follows from compassionate } \\
\text { care - chronic pain patients' experience } \\
\text { of integrative rehabilitation }\end{array}$ & $\begin{array}{l}\text { ARMAN; } \\
\text { HÖK. }\end{array}$ & 2016 & Suíça & N6 \\
\hline A8 & $\begin{array}{l}\text { The effectiveness of art therapy for } \\
\text { anxiety in adult women: A randomized } \\
\text { controlled trial }\end{array}$ & $\begin{array}{l}\text { ABBING } \\
\text { et al. }\end{array}$ & 2019 & $\begin{array}{l}\text { Holand } \\
\text { a }\end{array}$ & $\mathrm{N} 2$ \\
\hline A9 & $\begin{array}{l}\text { Rhythmical massage improves } \\
\text { autonomic nervous system function: a } \\
\text { single-blind randomised controlled trial }\end{array}$ & $\begin{array}{l}\text { SEIFER } \\
\mathrm{T} \text { et al. }\end{array}$ & 2018 & $\begin{array}{l}\text { Alema } \\
\text { nha }\end{array}$ & N2 \\
\hline & \multicolumn{5}{|c|}{ CATEGORIA 3- SAÚDE DA MULHER } \\
\hline $\begin{array}{l}\text { A1 } \\
0\end{array}$ & $\begin{array}{l}\text { Treating menopausal symptoms with a } \\
\text { complex remedy or placebo: } A \\
\text { randomized controlled trial }\end{array}$ & $\begin{array}{l}\text { VON } \\
\text { HAGEN } \\
\text { S et al. }\end{array}$ & 2012 & $\begin{array}{c}\text { Alema } \\
\text { nha }\end{array}$ & N2 \\
\hline $\begin{array}{l}\text { A1 } \\
1\end{array}$ & $\begin{array}{l}\text { Envelhecimento: combatê-lo ou } \\
\text { compreendê-lo? O climatério como } \\
\text { exemplo }\end{array}$ & GARDIN & 2017 & Brasil & N5 \\
\hline $\begin{array}{l}\text { A1 } \\
2\end{array}$ & $\begin{array}{l}\text { Personal perception and body } \\
\text { awareness of dysmenorrhea and the } \\
\text { effects of rhythmical massage therapy } \\
\text { and heart rate variability biofeedback-A } \\
\text { qualitative study in the context of a } \\
\text { randomized controlled trail }\end{array}$ & $\begin{array}{l}\text { BERGE } \\
\text { R et al. }\end{array}$ & 2019 & $\begin{array}{l}\text { Alema } \\
\text { nha }\end{array}$ & N2 \\
\hline & \multicolumn{5}{|c|}{ CATEGORIA 4- OBSTETRICIA } \\
\hline $\begin{array}{l}\text { A1 } \\
3\end{array}$ & $\begin{array}{l}\text { Differences in exosome populations in } \\
\text { human breast milk in relation to allergic } \\
\text { sensitization and lifestyle }\end{array}$ & $\begin{array}{l}\text { TORRE } \\
\text { GROSA } \\
\text { PARED } \\
\text { ES et al. }\end{array}$ & 2014 & Suíça & N4 \\
\hline $\begin{array}{l}\text { A1 } \\
4\end{array}$ & $\begin{array}{l}\text { Influence of alternative lifestyles on self- } \\
\text { reported body weight and health } \\
\text { characteristics in women }\end{array}$ & $\begin{array}{l}\text { SIMÖES } \\
\text {-WÜST } \\
\text { et al. }\end{array}$ & 2014 & $\begin{array}{c}\text { Suíça } \\
\text { Holand } \\
\text { a }\end{array}$ & N4 \\
\hline
\end{tabular}




\begin{tabular}{|c|c|c|c|c|c|}
\hline $\begin{array}{l}\text { A1 } \\
5\end{array}$ & $\begin{array}{l}\text { Sleep quality in pregnancy during } \\
\text { treatment with Bryophyllum pinnatum: } \\
\text { An observational study }\end{array}$ & $\begin{array}{l}\text { LAMBRI } \\
\text { GGER- } \\
\text { STEINE } \\
\text { R et al. }\end{array}$ & 2014 & Suíça & N4 \\
\hline $\begin{array}{l}\text { A1 } \\
6\end{array}$ & $\begin{array}{l}\text { A gestante na visão da antroposofia: } \\
\text { contribuições para um acompanhamento } \\
\text { psicológico integral }\end{array}$ & $\begin{array}{l}\text { HIOKA } \\
\text { et al. }\end{array}$ & 2015 & Brasil & N5 \\
\hline $\begin{array}{l}\text { A1 } \\
7\end{array}$ & $\begin{array}{l}\text { Anthroposophic lifestyle influences the } \\
\text { concentration of metals in placenta and } \\
\text { cord blood }\end{array}$ & $\begin{array}{l}\text { FAGER } \\
\text { STEDT } \\
\text { et al. }\end{array}$ & 2015 & Suécia & N4 \\
\hline $\begin{array}{l}\text { A1 } \\
8\end{array}$ & $\begin{array}{l}\text { Mães Waldorf: gestação e parto na } \\
\text { comunidade antroposófica }\end{array}$ & $\begin{array}{l}\text { BASTOS } \\
\text {; } \\
\text { PEREIR } \\
\text { A. }\end{array}$ & 2018 & Brasil & N6 \\
\hline $\begin{array}{l}\text { A1 } \\
9\end{array}$ & $\begin{array}{l}\text { Treatment of hyperemesis gravidarum } \\
\text { with anthroposophic complex therapy in } \\
3 \text { case reports }\end{array}$ & $\begin{array}{l}\text { KLOTER } \\
\text { et al. }\end{array}$ & 2019 & Suíça & N6 \\
\hline & \multicolumn{5}{|c|}{$\begin{array}{l}\text { CATEG } \\
\text { ORIA } 5 \\
\text { SISTEM } \\
\text { AS DE } \\
\text { SAÚDE }\end{array}$} \\
\hline $\begin{array}{l}\text { A2 } \\
0\end{array}$ & $\begin{array}{l}\text { Influence of Spirituality on Cool Down } \\
\text { Reactions, Work Engagement, and Life } \\
\text { Satisfaction in Anthroposophic Health } \\
\text { Care Professionals }\end{array}$ & $\begin{array}{l}\text { BÜSSIN } \\
\text { G et al. }\end{array}$ & 2015 & $\begin{array}{l}\text { Alema } \\
\text { nha } \\
\text { Suíça }\end{array}$ & N6 \\
\hline $\begin{array}{l}\text { A2 } \\
1\end{array}$ & $\begin{array}{l}\text { Usage of Complementary Medicine in } \\
\text { Switzerland: Results of the Swiss Health } \\
\text { Survey } 2012 \text { and Development Since } \\
2007\end{array}$ & $\begin{array}{l}\text { KLEIN et } \\
\quad \text { al. }\end{array}$ & 2015 & Suíça & N6 \\
\hline
\end{tabular}

fonte: elaborado pelas autoras (2022)

\section{DISCUSSÃO}

A Medicina Antroposófica é um sistema médico terapêutico complementar, que visa à compreensão do indivíduo e do seu processo saúde-doença, de forma integral, ao articular todas as suas dimensões: física, emocional, mental, espiritual e social (KIENLE et al., 2018). Em contraste ao modelo biomédico vigente, esta ciência amplia o entendimento da etiopatogenia, ao observar a influência de manifestações subjetivas no corpo físico e valorizar a complexidade destes aspectos, promovendo, assim, uma assistência em saúde mais profunda e 
humanizada. (TEIXEIRA, 2017).

A abordagem terapêutica, sob esta óptica, é sustentada pelos princípios da Salutogênese, que consiste em estimular as habilidades autocurativas do organismo e, também, de envolver o paciente no processo de melhoria da sua condição de saúde, promovendo o empoderamento do paciente por meio de ferramentas educativas e de autogestão. (BAARS; KOSTER; VERHOEF, 2017).

Na categoria 1 - Câncer de Mama, os estudos analisaram o aumento da qualidade de vida das mulheres, que utilizaram da Medicina Antroposófica, durante o tratamento de câncer de mama, revelando eficácia tanto nos sintomas físicos e psíquicos, quanto no que tange ao crescimento pessoal e a busca pelo sentido da vida. Sob a análise de aspectos subjetivos, que permeiam as suas experiências de vida, a qualificação das funções emocionais influi, também, na melhoria dos sintomas físicos vinculados ao tratamento quimioterápico.

Para CARLSSON et al. (2006), há o aumento na qualidade de vida e satisfação com a vida entre as mulheres tratadas com a terapia antroposófica quando comparada ao tratamento convencional, mesmo com a limitação quanto aos escores da avaliação de qualidade de vida feita no início do estudo, ou seja, mesmo com grupos não iguais.

Apesar da diminuição significativa na porcentagem de células CD8 do grupo sob terapêutica antroposófica, não houve alterações nos parâmetros imunológicos ao longo do tempo, em comparação com a terapia convencional (HAMRIN; ERNERUDH; ROSÉN, 2018). Porém, o estudo demonstra associação da diminuição dos sintomas de ansiedade e de sintomas físicos com a diminuição de células CD8, mas se torna necessário um estudo psiconeuroimunológico adicional. $\mathrm{O}$ autor relata também que um estudo randomizado seria preferível.

Para (ARMAN; BACKMAN, 2007), mulheres com tratamento antroposófico retratam estar mais direcionadas ao crescimento pessoal e à busca pelo sentido da vida, enquanto as mulheres com terapêutica convencional dedicam-se a atividades externas e físicas. Dessa forma, a adesão às $\mathrm{MTCl}$ e os cuidados antroposóficos pode ter sido benéfica para as mulheres em sua busca por um novo significado e de alcançar uma compreensão mais profunda da vida.

Na categoria 2 - Doenças crônicas e psicossomáticas, os estudos revelam a eficácia da terapêutica antroposófica no manejo de condições crônicas 
e psicossomáticas, como a ansiedade. Destaca-se também a alteração de outros parâmetros fisiológicos, ocasionando mudanças de estilo de vida que impactam na saúde da mulher de forma geral. Arman e Hök (2016) corroboram a importância e a necessidade de o profissional de saúde desenvolver e estimular as práticas de autocuidado e da gestão compartilhada para a manutenção de um novo modelo de saúde.

De acordo com HAMRE et al. (2009), as condições crônicas de saúde que mais prevalecem dentro do grupo de pacientes que fazem o uso de medicações antroposóficas são de origem psicológica, respiratória, musculoesquelética, neurológica e geniturinária. Para todos os critérios avaliados, demonstraram melhoria nos desfechos das doenças crônicas, ao longo dos seis primeiros meses de estudo, com o uso do medicamento antroposófico e foram similares nos pacientes sem 0 uso de medicamentos antroposóficos. Além disso, as medicações alopáticas geram mais efeitos adversos e, consequentemente, um impacto negativo na saúde dos pacientes em questão.

O estudo de (HAMRE et al., 2010) sobre tratamento antroposófico para enxaqueca foi avaliado numa população na qual $89 \%$ dos indivíduos eram mulheres. Dos desfechos analisados, a partir da linha de base do estudo, em relação aos follow-ups, houve melhoria significativa nos critérios de severidade da migrânea, classificação dos sintomas, funcionamento social, dor física, vitalidade e a autopercepção de mudança no estado de saúde. $78 \%$ dos pacientes apresentavam alguma outra doença crônica, corroborando com os dados apresentados por (HAMRE et al., 2009) em que as principais causas eram de origem psicocomportamental, geniturinária, musculoesquelética e, também, dermatológica.

Para ABBING et al. (2019), a terapêutica antroposófica é eficaz na redução da ansiedade, ocasionando um aumento na qualidade de vida e aumento das estratégias de regulação emocional. Essa terapêutica também mostrou melhora em pacientes com maior nível de ansiedade pré-tratamento, se contrapondo aos modelos de terapia convencional. Não está claro se os resultados são generalizáveis para mulheres menos motivadas com ansiedade, pois o estudo contou com exercícios artísticos com argila, desenho e pintura.

Para SEIFERT et al. (2018), é possível haver alterações no sistema 
cardiovascular logo após a prática terapêutica da massagem rítmica associada ao uso de óleo essencial. Dessa forma, validando o fato de que a sensibilidade barorreflexa é aumentada com a sua estimulação, além da possibilidade de que o óleo utilizado, óleo de jojoba, também possa ter um efeito olfativo significativo. Porém, após 24h, o efeito não era mais distinguível.

Compreendendo a saúde como um fenômeno complexo e sistêmico, os autores (ARMAN; HAMMARQVIST; KULLBERG, 2011) avaliaram as razões pelas quais os pacientes buscaram o atendimento em um hospital antroposófico, destacando-se a sua autopercepção por estarem mais positivos, as expectativas à respeito do cuidado que receberiam, os tratamentos antroposóficos e a visão holística do paciente. No que tange o impacto na continuidade do processo terapêutico e nos hábitos de vida, $86 \%$ dos pacientes reportaram dar continuidade ao tratamento após a alta hospitalar e $87 \%$ da amostra relatou uma mudança no padrão de humor e comportamento e, também, nas suas atividades de vida diária, como um ritmo de vida mais sereno e a prática de atividades físicas regulares.

Na categoria 3 - Saúde da mulher, os estudos abordam as experiências de vida da mulher, quando inseridas num contexto da medicina antroposófica. Entretanto, os autores indicam a falta de evidências necessárias acerca do uso de complexos medicamentos para o tratamento de sintomas do ciclo menstrual feminino (VON HAEGENS et al., 2012).

Conforme afirma Berger et al. (2019), as intervenções terapêuticas permitem o desenvolvimento de uma autoconsciência e consciência corporal que resultam em menos limitações na vida diária da mulher.

O período de transição hormonal experienciado pela mulher, da fase reprodutiva à pós-menopausa, pode manifestar uma série de alterações físicas como calorões, secura vaginal e ganho de peso, como também sintomas psicoemocionais como irrtabilidade, mudanças do humor, perda da libido, insônia e cansaço. GARDÍN (2017) refere que o uso de terapias hormonais bloqueia os processos naturais decorrentes do ciclo vital e, portanto, há a necessidade de encontrarmos novas perspectivas para o envelhecimento.

Na categoria 4 - Obstetrícia, evidenciou-se a influência dos estilos de vida da mulher durante a gestação tanto no desenvolvimento do feto, quanto na integridade da sua saúde física, mental e emocional. Ressalta-se também a 
necessidade de se recuperar a autonomia e fortalecer a autodeterminação frente aos desafios da maternidade. Para Paredes et al., 2014, a sensibilização materna e o estilo de vida podem influenciar no desenvolvimento de alergias na criança.

Em relação aos efeitos da gestação na saúde da mulher, as terapêuticas antroposóficas acarretam indivíduos com menor prevalência de sobrepeso, hipertensão, doença cardiovascular e hipertensão gestacional, e estas amamentam por mais tempo (Simões-Wuüst et al. 2014). Da mesma forma, segundo Kloterl et al. (2019), a terapêutica antroposófica induziu ao alívio de sintomas de mulheres com hiperêmese gravídica.

Para Lambrigger-Steiner et al., 2014, o uso de B. pinnatum é adequado para o tratamento de distúrbio do sono durante a gestação. Relatou melhora subjetiva na qualidade do sono, demonstrando diminuição do número médio de despertares à noite e dos problemas do sono, ao evitar a ocorrência de contrações leves durante a noite, além de diminuir os problemas de pele, como prurido. Nenhum efeito adverso grave foi relatado e a preparação foi bem tolerada.

Para Fagerstedt et al. (2015), mulheres com estilo de vida antroposófico têm maiores concentrações do elemento tóxico Cádmio (Cd), Cobalto (Co) e Chumbo $(\mathrm{Pb})$ no sangue materno e na placenta. $\mathrm{O}$ acúmulo de Cádmio provavelmente é resultado de uma dieta rica em alimentos derivados de plantas, dieta vegetariana, diminuição da concentração de ferro e/ ou ocupações maternas específicas, como arte, soldagem ou manuseio de metais. Dessa forma, dificulta o transporte de nutrientes e o desenvolvimento fetal por alterações na regulação hormonal. O estudo notou que o Cobalto está relacionado à Vitamina B12 e ao aumento da idade gestacional. A contribuição para a alteração no Chumbo não é conhecida. No entanto, é necessário ser elucidado quais os aspectos específicos do estilo de vida antroposófico para o aumento da exposição ao $\mathrm{Cd}$, Co e $\mathrm{Pb}$.

Para Hioka et al. (2015), é importante que o profissional envolvido no processo de cuidado de gestantes se aproprie de conteúdos multidisciplinares e variados. É valoroso que se envolvam, sejam empáticos e tratem com seriedade esse período, pois são esses profissionais que oferecerão o conhecimento e o esclarecimento necessários para o desenvolvimento do indivíduo, da gestante e futura mãe, a fim de recuperar a autonomia e fortalecer a autodeterminação da mulher, para que possam enfrentar de forma mais positiva essa nova fase da vida 
e as suas tarefas.

Para (BASTOS; PEREIRA, 2018) entre o equilíbrio emocional e o controle corporal encontra-se a construção do "caráter materno", articulando aspectos emocionais, corporais e espirituais, frente aos desafios da maternidade. Para isso, as orientações da Medicina Antroposófica exercem um grande desafio ao modelo biomédico, apesar de assumir uma postura ampliadora e não oponente à biomedicina.

Na categoria 5 - Sistemas de saúde, os estudos pontuam os impactos das PICS na vida dos profissionais de saúde e, consequentemente, na assistência prestada às mulheres. Os profissionais de saúde com atuação antroposófica, quando comparados aos que atuam na medicina convencional, aparentam ter estratégias de regulação emocional que colaboram diante das condições estressoras de trabalho, que são associadas a Síndrome de Burnout, e aumentando a sua qualidade de vida (Büssing et al., 2015).

Para Klein et al. (2015), os serviços de saúde estão cada vai mais utilizando métodos complementares como a homeopatia, naturopatia, osteopatia, fitoterapia e acupuntura. $25 \%$ da população local utilizou pelo menos uma Medicina Complementar e Integrativa nos últimos 12 meses e $26.7 \%$ de mulheres gestantes, que residem na Inglaterra, fizeram o uso de alguma MTCI durante a gestação, sendo as principais a fitoterapia e a homeopatia.

\section{CONSIDERAÇÕES}

As evidências científicas sobre $\mathrm{o}$ processo de empoderamento e autonomização da saúde da mulher destacam que a Medicina Antroposófica se revela como uma ferramenta ativa de mudança no paradigma da doença à potencialização da saúde. Caracteriza-se, portanto, como uma prática que contribui para o fortalecimento da autonomia e determinação da mulher nos seu processo de saúde-doença nas diferentes fases do ciclo vital.

A maioria dos autores destacam que a Medicina Antroposófica está associada ao aumento da qualidade de vida, no que tange ao psíquico, físico e emocional. Observou-se achados favoráveis à aplicação da terapia em diferentes períodos da vida da mulher, sobretudo em associação às terapias convencionais, e não como substituta ou uma imposição de nova estratégia terapêutica. 
Quanto às limitações da pesquisa, ressalta-se a necessidade de realização de estudos de maior nível de evidência. Isso possibilita assertividade e impacto da prática antroposófica, bem como qualificação da assistência em saúde.

No que se refere a contribuições, este estudo oferece bases teóricoconceituais importantes sobre as quais poderemos repensar determinadas condutas médicas, a partir da ampla circulação de saberes científicos, favorecendo uma mudança em que as mulheres retomam o protagonismo dos seus corpos e da sua saúde. Espera-se que as análises realizadas possam servir de subsídio para a capacitação multidisciplinar dos profissionais de saúde no que diz respeito à necessidade de ampliação das ferramentas terapêuticas para atendimento e o entendimento quanto aos direitos das mulheres. 


\section{REFERÊNCIAS}

ABBING, Annemarie. et al. The Effectiveness of Art Therapy for Anxiety in Adult Women: A Randomized Controlled Trial. Front Psychol. Holanda, vol. 29, n. 10, p. 1-14, 2019. Doi: https://doi.org/10.3389/fpsyg.2019.01203. Disponível em: https://www.ncbi.nlm.nih.gov/pmc/articles/PMC6549595/. Acesso em: 10 jul. 2020.

AGUIAR, Janaína Marques de; D'OLIVEIRA, Ana Flávia Pires Lucas. Violência institucional em maternidades públicas sob a ótica das usuárias. Interface Comunicação, Saúde, Educação, [S. I.], v. 15, n. 36, p. 79-92, 2010. Disponível em: https://doi.org/10.1590/s1414-32832010005000035. Acesso em: 05 jul. 2020. ANDRADE, João Tadeu de; COSTA, Liduina Farias Almeida da. Complementary Medicine in the SUS: Integrative practices in the perspective of medical Anthropology. Saude e Sociedade, [S. I.], v. 19, n. 3, p. 497-508, 2010. Disponível em: https://doi.org/10.1590/s0104-12902010000300003. Acesso em: 07 jul. 2020.

ARMAN, Maria; BACKMAN, M. A longitudinal study on women's experiences of life with breast cancer in anthroposophical (complementary) and conventional care. European journal of cancer care, Suécia, vol. 16, n. 5, p. 444-450, 2007. Doi: https://doi.org/10.1111/j.1365-2354.2006.00773.x Disponível em: https://onlinelibrary.wiley.com/doi/10.1111/j.1365-2354.2006.00773.x. Acesso em: 07 jul. 2020.

ARMAN Maria; HAMMARQVIST, Anne-Sofie; KULLBERG, Anna. Anthroposophic health care in Sweden - a patient evaluation. Complement Ther Clin Pract, Suécia, vol. 17, n. 3, p. 170-178, 2011. Doi: https://doi.org/10.1016/j.ctcp.2010.11.001. Disponível em: https://www.sciencedirect.com/science/article/abs/pii/S1744388110000952?via\% 3Dihub. Acesso em: 07 jul. 2020.

ARMAN, Maria; HÖK, Johanna. Self-care follows from compassionate care chronic pain patients' experience of integrative rehabilitation. Scand J Caring Sci, Suécia, vol. 30, n. 2, p. 374-381, 2016. Doi: https://doi.org/10.1111/scs.12258. Disponível em: https://onlinelibrary.wiley.com/doi/10.1111/scs.12258. Acesso em: 10 jul. 2020.

BAARS, Erik W.; KOSTER, Evi B.; VERHOEF, John. The Contribution of Anthroposophic Medicine to Self-Management: An Exploration of Concepts, Evidence, and Patient Perspectives. Complementary Medicine Research, [S. I.], v. 24, n. 4, p. 225-231, 2017. Disponível em: https://doi.org/10.1159/000478981. Acesso em: 10 jul. 2020.

BASTOS, Raquel Littério de; PEREIRA, Pedro Paulo Gomes. Mães Waldorf: gestação e parto na comunidade antroposófica. Interface - Comunicação, Saúde, Educação, Brasil, v. 22, n. 65, p. 505-516, 2018. Disponível em: https://doi.org/10.1590/1807-57622016.0651. Acesso em: 10 jul. 2020.

BASTOS, Raquel Littério de; PEREIRA, Pedro Paulo Gomes. Mães Waldorf: Gestação e parto na comunidade antroposófica. Interface: Communication, 
Health, Education, [S. I.], v. 22, n. 65, p. 505-516, 2018. Disponível em: https://doi.org/10.1590/1807-57622016.0651. Acesso em: 10 jul. 2020.

BERGER, B. et al. Personal perception and body awareness of dysmenorrhea and the effects of rhythmical massage therapy and heart rate variability biofeedback-A qualitative study in the context of a randomized controlled trail. Complement Ther Med, Alemanha, vol. 45, p. 280-288, $2019 . \quad$ Doi: https://doi.org/10.1016/j.ctim.2019.04.007. Disponível em: https://www.sciencedirect.com/science/article/abs/pii/S0965229918302966?via\% 3Dihub\#!. Acesso em: 11 jul. 2020.

BORGES, Maritza Rodrigues; MADEIRA, Lélia Maria; AZEVEDO, Vivian Mara Gonçalves de Oliveira. As práticas integrativas e complementares na atenção à saúde da mulher: uma estratégia de humanização da assistência no Hospital Sofia Fedlman. Rev. Min. Enferm. [S. I.], v. 15, n. 1, p. 105-113, 2011. Disponível em: https://pesquisa.bvsalud.org/portal/resource/pt/lil-600150. Acesso em: 11 jul. 2020.

BRASIL, Ministério da Saúde. Portaria n 971, de 03 de maio de 2006. Aprova a Política Nacional de Práticas Integrativas e Complementares (PICS) no Sistema Único de Saúde. Diário Oficial da União: Brasília, Ministério da Saúde, 2006. Disponível em: https://www.cff.org.br/userfiles/38\%20\%20BRASIL_\%20MINISTÉRIO\%20DA\%20SAÚDE_\%20Portaria\%20n%20971, \%20de\%2003\%20de\%20maio\%20de\%202006_pdf. Acesso em: 11 jul. 2020.

BÜSSING, Arndt. et al. Influence of spirituality on cool down reactions, work engagement, and life satisfaction in anthroposophic health care professionals. Evid Based Complement Alternat Med, Alemanha, v. 2015, 2015. Doi: https://doi.org/10.1155/2015/754814. Disponível em: https://www.hindawi.com/journals/ecam/2015/754814/. Acesso em: 05 jul. 2020.

CARLSSON, Marianne. et al. A Five-year Follow-up of Quality of Life in Women with Breast Cancer in Anthroposophic and Conventional Care. Evidence-based complementary and alternative medicine, Suécia, vol. 3, n. 4, p. 523-531, 2006. Doi: https://doi.org/10.1093/ecam/nel042. Disponível em: https://www.ncbi.nlm.nih.gov/pmc/articles/PMC1697754/pdf/nel042.pdf. Acesso em: 11 jul. 2020.

FAGERSTEDT, Sara. et al. Anthroposophic lifestyle influences the concentration of metals in placenta and cord blood. Environ Res, Suécia, v. 136, p. 88-96, 2015. Doi: $\quad$ https://doi.org/10.1016/j.envres.2014.08.044. Disponível em: https://www.sciencedirect.com/science/article/abs/pii/S0013935114003648?via\% 3Dihub. Acesso em: 07 jul. 2020.

GARDIN, Nilo E. Envelhecimento: combatê-lo ou compreendê-lo? O climatério como exemplo. Arte Médica Ampliada, Brasil, vol. 37, n. 1, p. 19-23, 2017. Disponível em: http://abmanacional.com.br/wp-content/uploads/2017/07/37-1Envelhecimento.pdf. Acesso em: 10 jul. 2020.

HAMRE, Harald J. et al. Outcome of anthroposophic medication therapy in chronic disease: a 12-month prospective cohort study. Drug Des Devel Ther, Alemanha, vol. $6, \quad$ n. 2, p. 25-37, 2009. Disponível em: 
https://pubmed.ncbi.nlm.nih.gov/19920891/. Acesso em: 07 jul. 2020.

HAMRE, Harald J. et al. Anthroposophic therapy for migraine: a two-year prospective cohort study in routine outpatient settings. Open Neurol J, Alemanha, vol. 4, p. 100 -110, 2010. Doi: https://doi.org/10.2174/1874205X01004010100. Disponível em: https://www.ncbi.nlm.nih.gov/pmc/articles/PMC3111720/. Acesso em: 07 jul. 2020.

HAMRIN, Elisabeth; ERNERUDH, Jan; ROSÉN, Anders. Immunological and Quality-of-Life Profiles in Women with Breast Cancer: Complementary versus Conventional Care. Complement Med Res, Suécia, vol. 25, n. 6, p. 391-397, 2018. Doi: https://doi.org/10.1159/000490049. Disponível em: http://liu.divaportal.org/smash/get/diva2:1276644/FULLTEXT01.pdf. Acesso em: 10 jul. 2020.

HIOKI, Sandra Akemi Okajima. et al. A gestante na visão da antroposofia: contribuições para um acompanhamento psicológico integral. Arte Médica Ampliada, Brasil, v. 35, n. 4, p. 158-165, 2015. Disponível em: http://abmanacional.com.br/arquivo/a151b751c3d5bd302b41801063bf728a3619f 95f-35-4-gestante.pdf. Acesso em: 05 jul. 2020.

KIENLE, Gunver S. et al. Anthroposophic Medicine: An Integrative Medical System Originating in Europe. Arte Médica Ampliada, [S. I.], v. 2, n. 6, p. 20-31, 2018. Disponível em: https://doi.org/10.7453/gahmj.2012.087. Acesso em: 10 jul. 2020.

KLEIN, Sabine D. et al. Usage of Complementary Medicine in Switzerland: Results of the Swiss Health Survey 2012 and Development Since 2007. PLoS One, Suíça, v. 10, n. 10, p.10, 2015. Doi: https://doi.org/10.1371 / journal.pone.0141985 /https://doi.org/10.1371 / journal.pone.0144676. Disponível em: https://www.ncbi.nlm.nih.gov/pmc/articles/PMC4626041/. Acesso em: 06 jul. 2020.

KLOTER, Evelyne. et al. Treatment of hyperemesis gravidarum with anthroposophic complex therapy in 3 case reports. Complement Ther Med, Suíça, v. 44, p. 14-17, 2019. Doi: https://doi.org/10.1016/j.ctim.2019.02.011. Disponível em:

https://www.sciencedirect.com/science/article/pii/S0965229918311580?via\%3Dih ub. Acesso em: 10 jul. 2020.

LAMBRIGGER-STEINER, C. et al. Sleep quality in pregnancy during treatment with Bryophyllum pinnatum: an observational study. Phytomedicine, Suíça, vol. 21, n. 5, p. 753-757, 2014. Doi: https://doi.org/10.1016/j.phymed.2013.11.003. Disponível em: https://www.sciencedirect.com/science/article/abs/pii/S0944711313004698?via\% 3Dihub. Acesso em: 05 jul. 2020.

LACERDA, Maria Ribeiro; COSTENARO, Regina Gema Santini. Metodologias da pesquisa para a enfermagem e saúde: da teoria à prática. 1. ed. Porto Alegre: Moriá, 2016. Acesso em: 20 dez. 2021.

MELNYK, Bernadette Mazurek; FINEOUT-OVERHOLT, Ellen (Ed.). Evidencebased practice in nursing \& healthcare: A guide to best practice. Lippincott Williams 
\& Wilkins, 2011. Acesso em: 20 dez. 2021.

PESSOA, Débora Luana Ribeiro. et al. O uso da aromaterapia na prática clínica e interprofissional. Research, Society and Development, v. 10, n. 3, p. 1-12, 2021. DOI: https://doi.org/10.33448/rsd-v10i3.13621. Disponível em: https://rsdjournal.org/index.php/rsd/article/view/13621. Acesso em: 15 jul. 2020.

PRÓ-REITORIA de Gestão de Pessoas e Qualidade de Vida (PROGEPE). Breve histórico sobre práticas integrativas e complementares em Saúde (PICS). Projeto Fluir com a Vida, PROGEPE: Universidade Federal de Pernambuco (UFPE), $2021 . \quad$ Disponível

https://www.ufpe.br/documents/2878072/2878531/Breve_histo 'rico-

pics+\%282\%29.pdf/9c22e090-1034-433b-af81-926daff2c2b2. Acesso em: 10 jul. 2020.

SEIFERT, Georg. et al. Rhythmical massage improves autonomic nervous system function: a single-blind randomised controlled trial. J Integr Med, Alemanha, vol. 16, n. 3, p. 172-177, 2018. Doi: https://doi.org/10.1016/j.joim.2018.03.002. Disponível em: https://www.sciencedirect.com/science/article/pii/S2095496418300372?via\%3Dih ub.

Acesso em: 05 jul. 2020.

SIMÕES-WÜST, Ana Paula. et al. Influence of alternative lifestyles on selfreported body weight and health characteristics in women. Eur J Public Health, Suíça, vol. 24, n. 2, p. 321-327, 2014. Doi: https://doi.org/10.1093/eurpub/ckt045. Disponível em: https://academic.oup.com/eurpub/article/24/2/321/447357. Acesso em: 05 jul. 2020.

TEIXEIRA, Marcus Zulian. Antropologia Médica Vitalista: uma ampliação ao entendimento do processo de adoecimento humano. Revista de Medicina, [S. I.], v. 96, n. 3, p. 145, 2017. Disponível em: https://doi.org/10.11606/issn.16799836.v96i3p145-158. Acesso em: 10 jul. 2020.

TESSER, Charles Dalcanale; BARROS, Nelson Filice de. Medicalização social e medicina alternativa e complementar: pluralização terapêutica do Sistema Único de Saúde. Revista de Saúde Pública, [S. I.], v. 42, n. 5, p. 914-920, 2008. Disponível em: https://doi.org/10.1590/s0034-89102008000500018. Acesso em: 06 jul. 2020.

TORREGROSA PAREDES P. et al. Differences in exosome populations in human breast milk in relation to allergic sensitization and lifestyle. Allergy, Suíça, vol. 69, n. 4, p. 463-471, 2014. Doi: https://doi.org/10.1111/all.12357. Disponível em: https://onlinelibrary.wiley.com/doi/10.1111/all.12357. Acesso em: 10 jul. 2020.

VON HAGENS, C. et al. Treating menopausal symptoms with a complex remedy or placebo: a randomized controlled trial. Climacteric, Alemanha, vol. 15, n. 4, p. 358-367, 2012. Doi: https://doi.org/10.3109/13697137.2011.597895. Disponível em:

https://www.tandfonline.com/doi/abs/10.3109/13697137.2011.597895?journalCo de=icmt20. Acesso em: 07 jul. 2020. 


\section{STUDI原S \\ PUBLICACOOES}

